\title{
The Chemotactic Response of Vibrio cholerae 0139 to The Mucilaginous Sheath of Blue-Green Algae Is Mediated by a Combination of Sheath Components
}

\author{
RAHMAN MD. MIZANUR ${ }^{1 *}$, MOHAMMAD SiRAJUl ISLAM ${ }^{2}$, SiRAJUl ISLAM KHAN ${ }^{1}$ and ZEAUR RAHIM ${ }^{2}$ \\ ${ }^{1}$ Department of Microbiology, University of Dhaka, Bangladesh \\ ${ }^{2}$ Environmental Microbiology Laboratory, International Centre for Diarrhoeal Disease Research, Bangladesh
}

(Received June 18, 2001—Accepted November 27, 2001)

In the aquatic environment, chemotaxis is one of the most important mechanisms of association between bacteria and other living biota. To investigate the mechanism of the chemotactic movement of Vibrio cholerae 0139 toward blue-green algae (BGA), the homogenates of four BGA species, Anabaena spp., A. variabilis, Nostoc spp. and Hapalosiphon spp., were examined by chemotaxis capillary assay. The assay revealed that $V$. cholerae 0139 swims toward the BGA with the highest chemotactic response observed in a 4\% solution of Anabaena spp. homogenate. The components of the mucilaginous sheath involved in this chemotactic response were examined. Major components were tested in a chemotaxis assay to determine the attractants present. A number of mucilaginous sheath-associated compounds, in particular free amino acids and carbohydrates, acted as chemoattractants for $V$. cholerae 0139 . Importantly, only when these attractants were combined into a single mixture were levels of chemotactic activity similar to those of Anabaena spp. It was revealed that $25^{\circ} \mathrm{C}$ and $1.7 \%$ salinity favored the chemotactic motility toward the homogenates of BGA. $V$. cholerae 01 was also attracted to homogenates of Anabaena spp.

Key words: chemotaxis, Vibrio cholerae, Blue-green algae

Cholera is one of three diseases subjected to International Health Regulations. This deadly disease is endemic in Bangladesh where cholera epidemics occur twice a year with a regular seasonality ${ }^{12)}$. During epidemics, Vibrio cholerae was isolated from surface water as well as from patients ${ }^{15,21)}$. The Indian subcontinent has experienced an epidemic of cholera caused by a newly recognized serotype of $V$. cholerae 0139 (synonym 'Bengal'), and this microorganism has emerged as the second etiological agent of this disease $^{3)}$. Recently, a 7th pandemic of cholera has been recorded: the causative agent of the last three pandemics was $V$. cholerae 01 . Vibrio cholerae 0139 was once proposed to be the causative agent of the 8 th pandemic of cholera ${ }^{3}$. Various reports have been published on the isolation of $V$.

\footnotetext{
* Corresponding author; Present address: 761-0701, Kagawa, Miki-cho, Ikenobe 2722-1, Abuniel Miki B-3, Japan. E-mail: mizan71@yahoo.com
}

cholerae 0139 from various aquatic and clinical sources in different parts of the world including Bangladesh ${ }^{19}$,22, 27, 28, ${ }^{30)}$. Huq et al. ${ }^{14)}$ demonstrated the coexistence of $V$. cholerae 0139 and 01 in plankton by a direct fluorescent antibody technique. We have reported earlier the occurance of culturable $V$. cholerae 0139 with a $c t x$ gene in various plants and animals of the aquatic environment in Bangladesh ${ }^{16)}$. The isolation of $V$. cholerae 0139 from its first appearance until its transient disappearence in 1994 and during its subsequent reemergence between 1995 and $1996^{8)}$ poses questions about possible reservoirs during interepidemic periods. Islam et al. ${ }^{18)}$ demonstrated the long-term survival and multiplication of $V$. cholerae 01 inside the mucilaginous sheath of a blue-green alga Anabaena variabilis. Although species information was not provided on the Gram-negative bacteria isolated from blue-green algae, mostly the isolates were Vibrio, Achromobacter, Aerobacter, Flavobacterium, Pseudomonas and Zoogloea ${ }^{6,23,29}$. On the other hand, $V$. 
cholerae 01 was detected inside the mucilaginous sheath of blue-green algae for up to 15 months in a laboratory-based study ${ }^{17)}$. This length of time is sufficient for the microorganism to persist during the interepidemic period. It was reported earlier in a field study that $V$. cholerae 01 was detected in the mucilaginous sheath of Anabeana spp. in 16 out of 24 plankton samples using an immunofluorescent technique ${ }^{17}$. This study indicated a mutual association between $V$. cholerae 01 and a blue-green alga Anabaena spp. in the natural environment. As both $V$. cholerae 01 and 0139 have almost the same pattern of survivality ${ }^{7,11,14)}$ in aquatic environmental samples, there is a possibility that the newly emerged $V$. cholerae 0139 can coexist with various blue-green algae.

Like other members of the Vibrio genus, $V$. cholerae 0139 exhibits rapid swimming motility in the aqueous milieu that is confirred by a polar flagellum. It has been reported that, chemotaxis plays a key role in the establishment and maintenance of cyanobacterial and bacterial associations $^{5,25)}$. Products of nitrogen fixation, excreted at heterocyst-vegetative cell junctions, were responsible for attracting various naturally occurring heterotrophic bacteria. Several reports have already been published regarding the extracellular materials liberated by different blue-green algal (BGA) species that are attached to the mucilaginous sheath $\left.^{9}, 10,13,20\right)$. The predominating products are amino acids such as glutamic acid, alanine, serine, threonine, glycine, tyrosine, valine, leucine, phenylalanine, and aspartic acid, and carbohydrates such as arabinose, xylose, rhamnose, galactose, glucose, and L-fucose. It seems likely that, to achieve an association with BGA, $V$. cholerae 0139 directs its passage toward the mucilaginous sheath by using chemotactic motility whereby components of the sheath act as chemoattractants.

The primary objective of this study was to measure the chemotactic response of $V$. cholerae 0139 to the homogenates of various blue-green algae and to investigate the basis of any response with respect to the composition of the mucilaginous sheath. The response of $V$. cholerae 01 to the homogenates of Anabaena spp. was also investigated. To our knowledge, this is the first report concerning the chemotactic activity of $V$. cholerae 0139 and 01 toward the homogenates of BGA species.

\section{Materials and Methods}

\section{Bacterial and blue-green algal strains}

An environmental isolate of $V$. cholerae 0139 (DWP341) obtained from duckweed ${ }^{16)}$, was provided by the
Environmental Laboratory, ICDDR,B and the purity and identity were reconfirmed by cultural, biochemical and serological testing. $V$. cholerae 01 , isolated from water, was maintained in the laboratory. The blue-green algae used in this study were Anabaena spp., A. variabilis, Nostoc spp. and Hapalosiphon spp. All the BGA species were obtained from the Environmental Microbiology Laboratory, ICDDR,B.

\section{Media and growth conditions}

$V$. cholerae serotype 0139 and 01 were maintained in

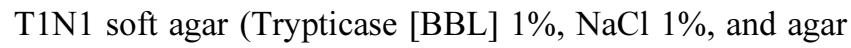
$0.8 \%$ ) in vials containing paraffin oil at room temperature and grown at $37^{\circ} \mathrm{C}$ in trypticase soy broth (TSB) (BBL). The vibrio selective medium used was thiosulfate citrate bile sucrose (TCBS) agar (Difco Laboratories, USA). The soft agar used for assaying the motility of Vibrio strains was TSB with $0.3 \%$ agar. All the BGA strains were grown and maintained in pure cultures on BG-11 medium $^{31)}$ at room temperature. The chemotaxis and washing medium used in this study was phosphate-buffered saline (PBS) ( $\mathrm{pH} \mathrm{7.4,}$ $\mathrm{NaCl}, 0.8 \%$ ).

\section{Chemicals and water}

All compounds tested in the chemotaxis assay were purchased from Sigma Chemical Co. (USA). Glass-distilled deionized water was used in all experiments.

\section{Preparation of homogenates of blue-green algae}

A defined amount $(0.05 \mathrm{~g})$ of each species of BGA in BG-11 medium was removed with a sterile spatula from the culture flask, washed three times with previously autoclaved PBS, and finally mixed with $1 \mathrm{ml}$ of PBS to make a $5 \%$ suspension. It was then homogenized using a glassmade hand homogenizer and finally with an ultrasonicator. This homogenized solution was then diluted to make the desired solution for chemotaxis assay.

\section{Chemotaxis assay}

A previously described quantitative capillary assay ${ }^{2)}$ was modified and used to measure the chemotaxis of $V$. choleare 0139. Strain 0139 was grown overnight in TSB. The overnight culture was diluted 10 -fold in TSB and reincubated for up to $4 \mathrm{~h}$ to maximize the number of motile cells before proceeding to the assay. The bacterial cells were harvested at $8000 \times \mathrm{g}$ for $5 \mathrm{~min}$ and resuspended in an equal volume of PBS. This washing step was repeated three times, and the final suspension was made in chemotaxis medium (PBS) to give an estimated cell density of $10^{10} \mathrm{cfu} / \mathrm{ml}$. Serial dilutions 
of the bacterial suspension in PBS were used to obtain counts of viable and culturable cells (colony forming units). A bacterial suspension of about $10^{7} / \mathrm{ml}$ viable cells was dispensed in 200- $\mu$ l aliquots into a 1-cc syringe ([B-D]R Brand, Becton Dickinsion medical products Ptc. Ltd. Singapore). A $1-\mu$ l capillary tube (Drummond Scientific Co.), heat sealed at one end and containing the substrate (in PBS) to be tested in half the length of the tube, was inserted horizontally into the syringe to approximately $1.0 \mathrm{~cm}$ below the surface of the bacterial solution. After incubation for 15, 30, $45,60,75$ and $90 \mathrm{~min}$ at room temperature, the capillaries were removed, the exterior was rinsed with a thin stream of PBS, and the contents were expelled into a specified amount of chemotaxis medium. A ten-fold dilution was made in the same medium and plated on TCBS agar. Counts of viable cells were then made. In each experiment, substrates were simultaneously tested in triplicate, and control capillaries containing PBS were included. The chemotactic activity of a particular substrate was expressed as percent accumulation (PA), i.e., the ratio of bacterial cells accumulated in substrate-containing capillaries to those contained in the $200-\mu 1$ suspension in the syringe multiplied by 100 .

Influence of temperature and salinity on the chemotaxis of $V$. choleare 0139

The effect of temperature and salinity on the chemotactic response of $V$. choleare 0139 to the homogenates of BGA was evaluated as follows. A bacterial culture was prepared as described above, to obtain a suspension with approximately $10^{7}$ bacterial cells per $\mathrm{ml}$. The bacterial suspension was prepared in a sea-water-based medium with different salinities and incubated for $8 \mathrm{~h}$ at different temperatures. The homogenates of different BGA species were prepared in 1, 1.7 and $3.5 \%$ salt solutions and the chemotaxis assay was performed as described above at 15,20 and $25^{\circ} \mathrm{C}$ for 90 min. The different salinity values were achieved by diluting filtered raw seawater (salinity 3.5\%) with filtered deionized water.

\section{Results}

Chemotactic response of $V$. choleare 0139 to the homogenates of different blue green algae

A large accumulation of $V$. cholerae $\mathrm{O} 139$ in the capillaries containing the homogenates of Anabaena spp. compared with the control capillary containing PBS was observed (Fig. 1). The response was highest at $4.0 \%$ homogenate and the maximum number of bacteria was found at $90 \mathrm{~min}$. Although the relative levels were low, the same pattern of

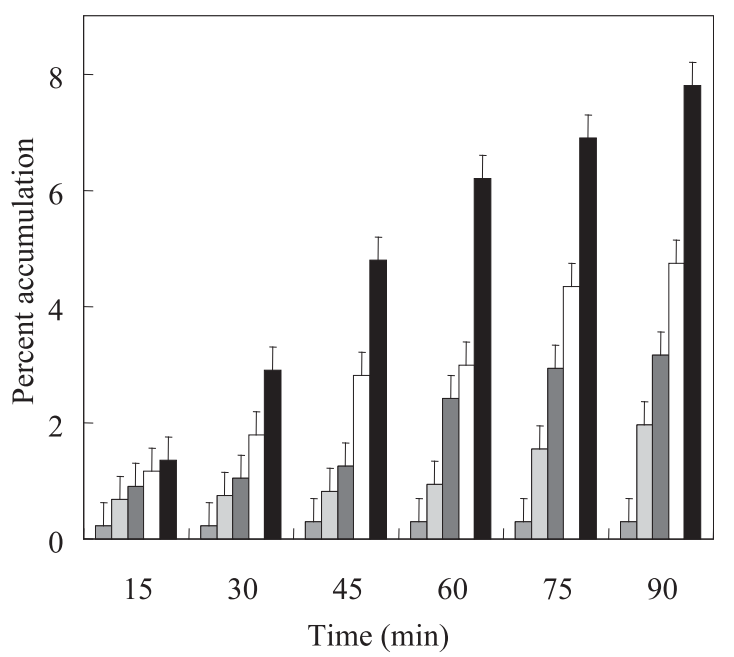

Fig. 1. Chemotaxis of $V$. cholerae 0139 toward the homogenates of Anabaena spp. Error bars indicate standard deviation. Symbols: $\square$, control; $\square, 0.5 \% ; \square, 1.0 \% ; \square, 2.0 \%$; $\square, 4 \%$.

accumulation was observed with homogenates of Nostoc spp. (Fig. 2). On the other hand, very young cultures of $A$. variabilis without a well-formed mucilaginous sheath showed a weaker chemotactic response towards the $V$. cholerae O139 (Fig. 3), and the highest chemotactic response was observed at $0.5 \%$ homogenate of Hapalosiphon spp. after the $4 \%$ suspension (Fig. 4). The 'threshold value', the lowest concentration of attractant that gives an accumulation in the capillary greater than that obtained in the absence of attractant, for each species was $0.1 \%$ for both Anabaena spp., Nostoc spp. and A. variabilis and $0.01 \%$ for Hapalosiphon spp. (Fig. 5).

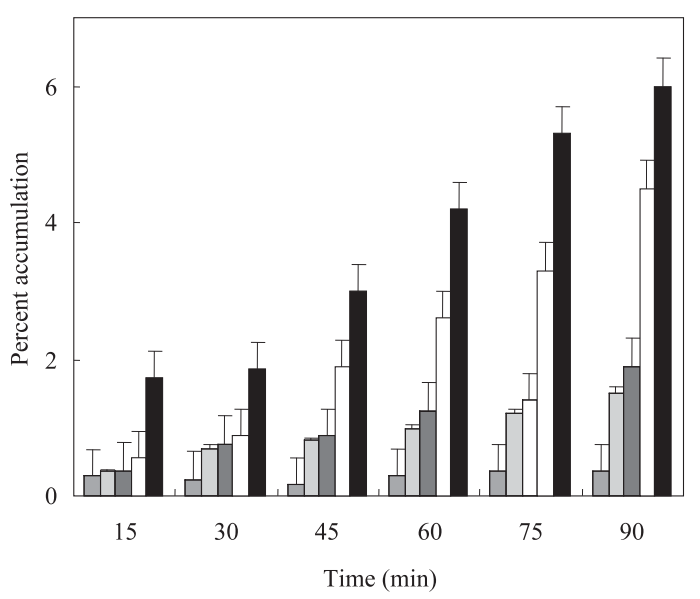

Fig. 2. Chemotaxis of $V$. cholerae 0139 toward the homogenates of Nostoc spp. Error bars indicate standard deviation. Symbols: $\square$, control; $\square, 0.5 \% ; \square, 1.0 \% ; \square, 2.0 \% ; \square, 4 \%$. 


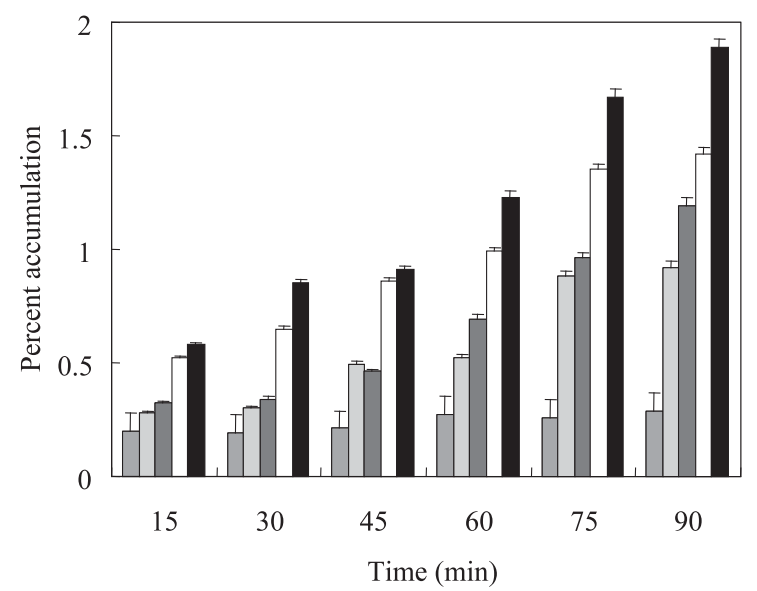

Fig. 3. Chemotaxis of $V$. cholerae 0139 toward the homogenates of A. variabilis. Error bars indicate standard deviation. Symbols: $\square$, control; $\square, 0.5 \% ; \square, 1.0 \% ; \square, 2.0 \% ; \square, 4 \%$.

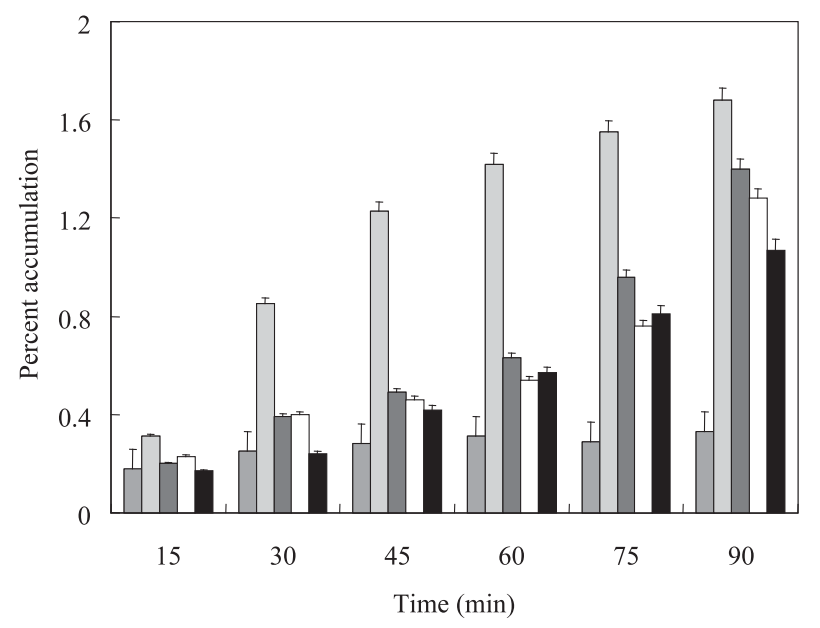

Fig. 4. Chemotaxis of $V$. cholerae 0139 toward the homogenates of Hapalosiphon spp. Error bars indicate standard deviation. Symbols: $\square$, control; $\square, 0.5 \% ; \square, 1.0 \% ; \square, 2.0 \% ; \square, 4 \%$.

\section{Chemotactic response towards individual components}

Commercial preparations of compounds identified in the extracellular material of $\mathrm{BGA}^{9,10,13,20)}$ were individually tested in the chemotaxis assay at concentrations of $0.1,1.0$, 5.0 and $10 \mathrm{mM}$. The chemotactic response to individual components at a concentration of $1.0 \mathrm{mM}$ is illustrated in Table 1. Compounds for which the percent accumulation was consistently less than $0.4 \%$ were not considered as significant chemoattractants. Of the compounds examined for chemotaxis of $V$. choleare 0139 , the amino acids serine, threonine, glycine, glutamic acid, leucine, isoleucine and histidine, and the carbohydrates fucose, glucose, mannose,

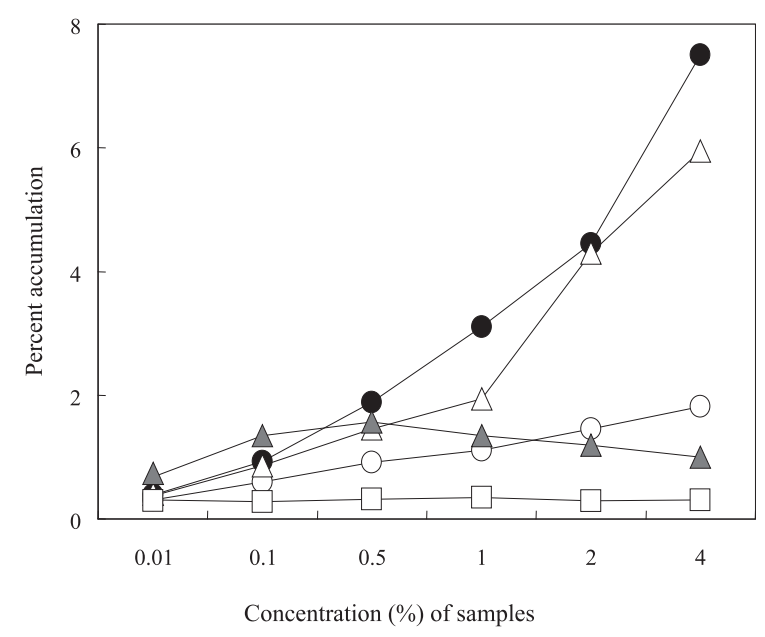

Fig. 5. Response of $V$. cholerae 0139 to different concentrations of homogenates of BGA species. Symbols: - Anabaena spp.; $\triangle$, Nostoc spp.; $\bigcirc$, A. variabilis; $\boldsymbol{\Lambda}$, Hapalosiphon spp.; $\square$, control. A number of concentrations (ranging from 0.01 to $4.0 \%$ ) of homogenates of BGA species were tested in a chemotaxis assay for 90 min to determine the threshold value.

ribose, arabinose, and xylose, displayed significant responses above $0.4 \%$ and thus represented chemoattractants for $V$. cholerae 0139 (Table 1). Commercial preparations of the individual components identified in the extracellular materials of BGA were each combined at a concentration of 0.1 mM. All BGA-associated attractants and, apparently, all BGA components with an individual percent accumulation below $0.4 \%$ were combined at the above concentrations, and the resulting mixtures were examined in the chemotaxis assay to compare their activities with the activity of the $4.0 \%$ Anabaena spp. homogenates. The combination of each of the BGA attractants at a concentration of 0.1 $\mathrm{mM}$ produced a mixture with $94 \%$ of the activity of the Anabaena spp. homogenates (Fig. 6). In contrast, mixtures containing tested BGA components with an individual percent accumulation below $0.4 \%$ possessed less than $5 \%$ of the activity of the Anabaena spp. homogenates (Fig. 6).

Effects of temperature and salinity on the chemotaxis of V. cholerae 0139

Various temperatures and salinities were selected to evaluate their effects on the chemotaxis of $V$. cholerae 0139. The minimal values of chemotactic response toward the homogenates of BGA were observed at the lowest temperature $\left(15^{\circ} \mathrm{C}\right)$ and most extreme salinity $(3.5 \%)$. The numbers of bacteria attracted to the homogenates of BGA in the different assay conditions are given in Table 2. The highest number of cells of $V$. cholerae 0139 was obtained at a salinity 
Table 1. Chemotactic response of $V$. cholerae 0139 to amino acids and carbohydrates reported in blue-green algae ${ }^{\text {a }}$.

\begin{tabular}{cc}
\hline \multicolumn{1}{c}{ Chemicals } & Percent accumulation $^{\mathrm{b}}$ \\
\hline Amino acids & 1.20 \\
Glutamic acid & 0.30 \\
Alanin & 4.80 \\
Threonine & 2.80 \\
Glycine & 0.40 \\
Tyrosine & 0.40 \\
Valine & 1.30 \\
Leucine & 0.30 \\
Phenylalanine & 0.40 \\
Aspertic acid & 3.80 \\
Isoleucine & 3.70 \\
Histidine & 5.0 \\
Serine & \\
Carbohydrates & 1.80 \\
L-Fucose & 0.30 \\
D-Galactose & 5.10 \\
D-Glucose & 4.80 \\
D-Mannose & 3.40 \\
D-Ribose & 3.90 \\
D-Arabinose & 3.10 \\
D-Xylose & 0.30 \\
Rhamnose & 0.31 \\
Galacturonic acid & 0.33 \\
Glucuronoc acid & 0.30 \\
Gluconic acid & 0.30 \\
Control & 10 \\
\hline chora & \\
\hline
\end{tabular}

a The chemotactic response of $V$. cholerae 0139 to individual amino acids and carbohydrates at $0.1,1.0 .5 .0$ and $10 \mathrm{mM}$ concentrations was measured in a capillary assay for $90 \mathrm{~min}$ and is expressed in terms of percent accumulation.

${ }^{\mathrm{b}}$ Accumulation for each substrate when present at a concentration of $1.0 \mathrm{mM}$ is illustrated.

of $1.7 \%$ and temperature of $25^{\circ} \mathrm{C}$ for the homogenate of Anabaena spp. The percent accumulation obtained in experiments in which the strains were preincubated for $8 \mathrm{~h}$ in sea water, a nutrient-deficient medium, were lower than that when the strains assayed were grown in TSB.

Chemotactic response of $V$. cholerae 01 to the homogenates of Anabaena spp.

The chemotactic response of $V$. cholerae 01 to the homogenates of Anabaena spp. was assayed. The homogenates at a concentration of $4 \%$ induced maximum accumulation at $90 \mathrm{~min}$ in the capillaries (Fig. 7). The response was a bit lower than that obtained with $V$. cholerae 0139 using the

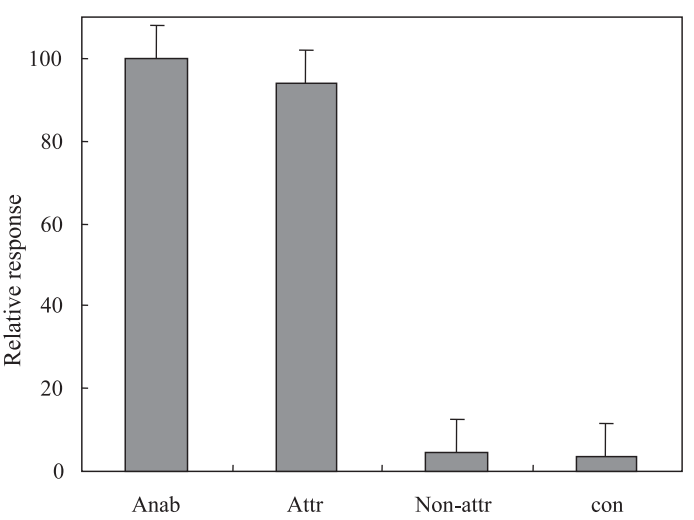

Fig. 6. Chemotactic activity of combinations of major chemical components reported in the mucilaginous sheath of BGA. Error bars indicate standard deviation. Symbols: Anab, Anabaena spp. homogenates; Attr: Combined chemicals (positive attractants); Non-attr: Combined chemicals (non-attractants); con, Control (PBS). The chemotaxis assay for each component was performed at room temperature for $90 \mathrm{~min}$.

same concentration of Anabaena spp. homogenates as shown in Fig. 1.

\section{Discussion}

In this study, the chemotactic responses of $V$. cholerae O139 to the extracts of four BGA species were tested in a chemotaxis capillary assay. Among the four algal homogenates examined, the extracts of Anabaena spp. were the best chemoattractants for $V$. cholerae $\mathrm{O} 139$ (Fig 1). Except for Hapalosiphon spp., the highest chemotaxis was observed with the $4.0 \%$ suspension regardless of the BGA species examined. For Hapalosiphon spp., 0.5\% homogenate showed better chemoattraction for $V$. cholerae $\mathrm{O} 139$. The reason behind this is not clear, but it might be assumed that inhibitory components exist in the extracts of Hapalosiphon spp. which are more effective at high concentrations. Therefore, these compounds might inhibit the bacterial chemotaxis. Among the BGA homogenates studied, those of $A$. variabilis were the weakest chemoattractors for $V$. cholerae O139 (Fig. 3). This might be because the rudimentary sheath of $A$. variabilis has much smaller amounts of excretory products which could not attract much vibrios. It has been reported that the excretory metabolites of BGA remain attached to the mucilaginous sheath and further act as chemoattractors for the vibrios ${ }^{26)}$. Furthermore, the extent of the excretion was greater in older cultures with a mature mucilaginous sheath ${ }^{5)}$. These investigations were consistent with our findings.

The chemical components of the algal extracellular prod- 


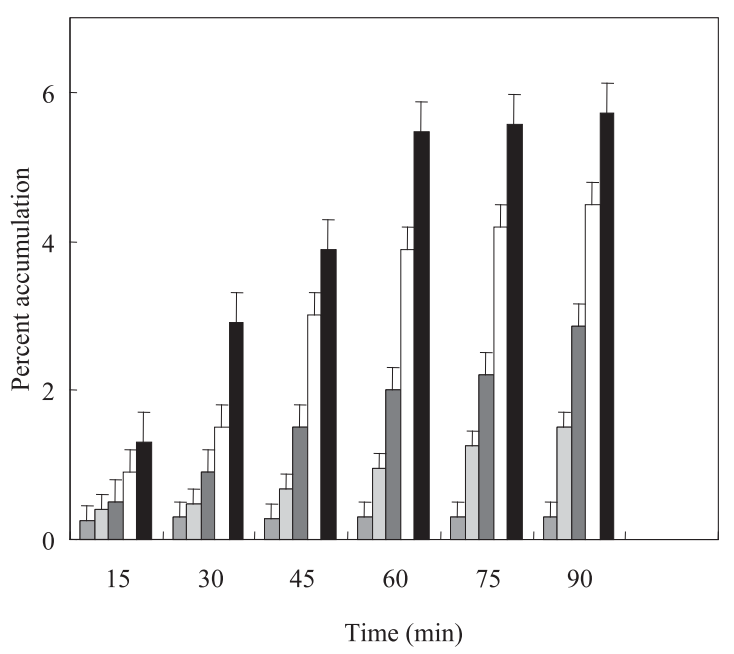

Fig. 7. Chemotaxis of $V$. cholerae 01 toward the homogenates of Anabaena spp. Error bars indicate standard deviation. Symbols: $\square$, control; $\square, 0.5 \% ; \square, 1.0 \% ; \square, 2.0 \% ; \square, 4 \%$.

ucts are the likely sources of microbial nutrients. As they may constitute a significant portion of primary production, such compounds are indeed of potential significance in microbial food chains. Experiments suggested that the extracellular materials are released as products of cell metabolism ${ }^{24)}$. However, whatever the source of the extracellular products, our data indicate that $V$. cholerae $\mathrm{O} 139$ is a chemotactic for homogenates of BGA. This response was invariably greatest for homogenates of old algal cultures (Fig. 1 and 2), implying that the release of extracellular carbon is ecologically most important during the later stages of a plankton bloom.

The major commercial preparations of the compounds in the extracellular material of the BGA were tested individually for chemotactic activity with respect to $V$. cholerae 0139. The results are summerized in Table 1. By combining each of the major components into a single mixture, it was possible to reconstitute a high level of chemotactic activity. In contrast, when presented alone, none of the sheath com- ponents possessed chemotactic activity equivalent to that of Anabaena spp. homogenates. Thus, rather than containing a single potent chemoattractant, it appears that the mucilaginous sheath of BGA consists of a range of chemotattractants which contribute to the overall activity of the sample.

An effect of environmental factors such as temperature and salinity, on the chemotactic activity of $V$. cholerae 0139 toward the homogenates of BGA has been observed. The highest degree of chemotaxis regardless of the BGA species was observed at $25^{\circ} \mathrm{C}$ and $1.7 \%$ salinity. It could be assumed that, at this temperature, the synthesis of the chemotactic machinery is favored. It was reported that the synthesis of lateral flagella of Vibrio spp. was maximal at a temperature and salinity of between 15 and $37^{\circ} \mathrm{C}$ and 1 to $2 \%{ }^{4)}$, respectively, which ultimately supports our findings. $V$. cholerae 01 also showed a chemotactic response toward the homohgenates of Anabaena spp. (Fig. 7).

As shown in Figs. 1-4 and 7, levels of both $V$. cholerae 0139 and 01 were insignificant in control capillaries compared to capillaries containing homogenates of BGA species in varying concentrations. If bacteria entered passively by convection and not by chemotaxis, similar percentages of bacterial cells could be expected in all capillaries including control capillaries. Due to chemotactic movement, significant numbers of $V$. cholerae accumulated in capillaries containing various concentrations of homogenates. It was reported that an attractant must be present in the capillary in order to ensure chemotaxis of $E$. coli ${ }^{2}$, which supports our findings. As shown in Table 1, the presence of certain chemicals, especially amino acids and carbohydrates, induced chemotaxis of $V$. cholerae 0139 , where as, other closely related chemicals could not produce any positive effect. If other factors, such as osmotic flow, were effective, the same effect would be observed for all the chemicals examined. It has been reported that bacterial cells have specific chemoreceptors for specific chemicals by which they can sense the presence and move toward $\mathrm{it}^{1}{ }^{1}$. For this reason, $V$. cholerae 0139 accumulated in large numbers in the capi-

Table 2. Influence of temperature and salinity on the chemotactic activity of $V$. cholerae 0139 toward homogenates of various BGA species.

\begin{tabular}{|c|c|c|c|c|c|c|c|c|c|}
\hline \multirow{3}{*}{ BGA species } & \multicolumn{9}{|c|}{ Percent accumulation at indicated temperature and salinity } \\
\hline & \multicolumn{3}{|c|}{$15^{\circ} \mathrm{C}$} & \multicolumn{3}{|c|}{$20^{\circ} \mathrm{C}$} & \multicolumn{3}{|c|}{$25^{\circ} \mathrm{C}$} \\
\hline & $1.0 \%$ & $1.7 \%$ & $3.5 \%$ & $1.0 \%$ & $1.7 \%$ & $3.5 \%$ & $1.0 \%$ & $1.7 \%$ & $3.5 \%$ \\
\hline Anabaena spp. & 2.8 & 4.1 & 1.9 & 5.3 & 6.2 & 1.9 & 6.0 & 7.0 & 2.1 \\
\hline A. variabilis & 0.8 & 1.2 & 0.6 & 1.2 & 1.5 & 0.9 & 1.8 & 2.0 & 0.9 \\
\hline Nostoc spp. & 2.0 & 3.0 & 1.5 & 3.1 & 4.3 & 2.0 & 4.0 & 5.8 & 1.8 \\
\hline Hapalosiphon spp. & 1.2 & 2.0 & 0.8 & 1.8 & 2.5 & 1.2 & 2.0 & 3.0 & 1.5 \\
\hline
\end{tabular}


llary containing D-glucose, where as they could not sense and therefore, could not move to the rhamnose-containing capillary (Table 1). In another study, carried out with a motile, wild-type E. coli $\mathrm{K} 12$ and mutants lacking aspartate and galactose receptors, it was reported that the mutants did not accumulate in the capillaries containing aspartate and galactose respectively beyond the level found in control capillaries $^{1}$. Therefore, it has been hypothesized that nonmotile $E$. coli has no chemotactic ability and motile but nonchemotactic mutants showed no increased accumulation in the capillaries containing attractants ${ }^{1,2}$. Our experimental evidence along with previous findings described above, strongly support that the accumulations of $V$. cholerae 01 and 0139 in the capillaries containing various attractants were mediated only by chemotaxis.

In this study it was not possible to investigate the concentration of homogenates beyond $4.0 \%$, as above this they could not be dispensed inside the capillaries. For statistical analysis, a t test was used to examine the results at a $5 \%$ level of significance. In conclusion, we have revealed that chemotactic motility might have been influenced by extracellular products of BGA that mediate the movement of $V$. cholerae $\mathrm{O} 139$ and 01 towards the mucilaginous sheath of BGA. Since, both $V$. cholerae 0139 and 01 were chemotactic to the homogenates of BGA, and we have shown earlier the long-term survival and multiplication of $V$. cholerae 01 inside the mucilaginous sheath of $A$. variabilis ${ }^{18)}$, the possibility exists that during interepidemic periods, epidemic strains of $V$. cholerae $\mathrm{O} 1$ and $\mathrm{O} 139$ use blue-green algae as a sanctuary to survive the adverse environmental conditions that favor the spread of the cholera disease during an epidemic.

\section{Acknowledgements}

This research was supported by the International Centre for Diarrhoeal Disease Research, Bangladesh (ICDDR,B). The ICDDR,B is supported by countries and agencies which share its concern for the health problems of developing nations.

\section{References}

1) Adler, J. 1969. Chemoreceptor in bacteria. Science 166: 15881597.

2) Adler, J. 1973. A method for measuring chemotaxis and use of the method to determine optimum conditions for chemotaxis by Escherichia coli. J. Gen. Microbiol. 74: 77-91.

3) Albert, M.J. 1994. Minireview: Vibrio cholerae 0139 Bengal. J. Clin. Micrbiol. 32: 2345-2349.
4) Belas, M.R. and R.R. Colwell. 1982. Adsorption kinetics of laterally and polarly flagellated Vibrio. J. Bacteriol. 151: 1568-1580.

5) Bell, W. and R. Mitchell. 1972. Chemotactic and growth response of marine bacteria to algal extracellular products. Biol. Bull. 143: 265-277.

6) Caldwell, D.E., S.J. Caldwell. 1978. A Zoogloea sp. associated with blooms of Anabaena flos-aquae. Can. J. Microbiol. 24: 922 931.

7) Colwell, R.R., R.J. Seidler, S.W. Kaper, S. Joseph, H. Garyes, M. D. Lockman, H. Bradford, N. Roberts, I. Huq and A. Huq. 1981. Occurance Vibrio cholerae 01 in Maryland, and Lousiana estuaries. Appl. Environ. Microbiol. 41: 555-558.

8) Faruque, S.M., K.M. Ahmed, A.K. Siddique, K. Zaman, A.R.M.A. Alim and M.J. Albert. 1997. Molecular analysis of toxigenic Vibrio cholerae 0139 Bengal strains isolated in Bangladesh between 1993 and 1996. J. Clin. Microbiol. 35: 2299-2306.

9) Fogg, G.E. 1952. The production of extracellular nitrogenous substances by a blue-green algae. Proc. R. Soc. London. Ser-B. 139: 372-397.

10) Fogg, G.E. and H. Pattnaik. 1966. The release of extracellular nitrogenous products by Westiellopsis prolifica. Phykos. 5: 5867.

11) Ghosh, A.R., H. Koley, D.De, S. Garg, M.K. Bhattacharya, S.K. Bhattacharya, B. Manna, G.B. Nair, T. Shimada, T. Takeda and Y. Takeda. 1994. Incidence and toxigenecity of Vibrio cholerae in fresh water lake during the epidemic cholera caused by serogroup 0139 Bengal in Calcutta, India. FEMS Microbiol. Ecol. 14: 285-291.

12) Glass, R.I., S. Becker, M.I. Huq, B.J. Stoll, M.U. Khan, M.H. Merson, J.V. Lee and R.E. Black. 1982. Epidemic cholera in rural Bangladesh, 1966-1980. Am. J. Epidemiol. 116: 959-970.

13) Hellebust, J.A. 1974. Algal physiology and biochemistry. Bot. Monogr. 10: 838-863.

14) Huq, A., R.R. Colwell, M.A.R. Chowdhury, B. Xu, S.M. Moniruzzaman, M.S. Islam, M. Yunus and M.J. Albert. 1995. Coexistence of Vibrio cholerae 01 and 0139 Bengal in plankton in Bangladesh. Lancet 345: 1249.

15) Huq, A., R.R. Rahman, A.Ali, M.A.R. Chowdhury, S. Parveen, D.A. Sack and E.R. Cohen. 1990. Detection of Vibrio cholerae 01 in the aquatic environment by fluorescent-monoclonal antibody and culture methods. Appl. Environ. Microbiol. 56: 23702373.

16) Islam M.S., M.J. Alam, A. Begum, Z. Rahim, A. Felsenstein and M.J. Albert. 1996. Occurrence and distribution of culturable Vibrio cholerae 0139 with $c t x$ gene in various components of the aquatic environment in Bangladesh. Trans. Royal Soc. Trop. Med. Hyg. 90: 128.

17) Islam, M.S., B.S. Drasar and R.S. Bradley. 1994. Review article: Probable role of blue-green algae in maintaining endemicity and seasonality in Bangladesh: a hypothesis. J. Diarrhoeal Dis. Res. 12: $245-256$.

18) Islam, M.S., D.S. Drasar and D.J. Bradley. 1990. Long term persistence of toxigenic Vibrio cholerae 01 in the mucilaginous sheath of a blue-green alga, Anabaena variabilis. J. Trop. Med. Hyg. 93: 133-139.

19) Islam, M.S., M.K. Hasan, M.A. Miah, M. Yunus, K. Zaman and M.J. Albert. 1994. Isolation of Vibrio cholerae 0139 synonym Bengal from aquatic environment in Bangladesh: implication for disease transmission. Appl. Environ. Microbiol. 60: 1684-1686. 
20) Jones, J.K.N., L. Hough and W.H. Wadman. 1952. An investigation of the polysaccharide components of certain freshwater algae. J. Chem. Soc. 3: 3393-3399.

21) Khan, M.U., M. Shahidullah, M.S. Haque and W.U. Ahmed. 1984. Presence of vibrios in surface water and their relation with cholera in community. Trop. Geor. Med. 36: 335-340.

22) Lee, S.H., S.T. Lai, J.Y. Lai and N.K. Leung. 1996. Resurgence of cholera in Hong Kong. Epidemiol. Infect. 117: 43-49.

23) Martin, Y.P. and M.A. Bianchi. 1980. Structure, diversity and catabolic potentialities of aerobic heterotrophic bacterial populations associated with continuous cultures of natural marine phytoplankton. Microbiol. Ecol. 5: 265-279.

24) Nalewajko C. and D.R.S. Lean. 1972. Growth and excretion in planktonic algae and bacteria. J. Phycol. 8: 361-366.

25) Pearl, H.W. and K.K. Gallucci. 1985. Role of chemotaxis in establishing a specific nitrogen-fixing cyanobacterial-bacterial association. Science 227: 647-649.
26) Pearl, H.W. 1978. Role of hetrotrophic bacteria in promoting N2 fixation by Anabaena in aquatic habitats. Microbiol. Ecol. 4: 215-231.

27) Pokhrel, B. M. and T. Kubo. 1996. Out breaks of cholera in Nepal. Southeast Asian J. Trop. Med. Pub. Health 27: 574-579.

28) Sengupta, T.K., D.K. Sengupta, G.B. Nair and A.C. Ghose. 1994. Epidemic isolates of Vibrio cholerae 0139 express antigenically distinct types of colonization pili. FEMS Microbiol. Lett. 118: 265-271.

29) Simidu, U., K. Ashino and E. Kaneko. 1971. Bacterial flora of phyto- and zoo- plankton in the inshore water of Japan. Can. J. Microbiol. 17: 1157-1160.

30) Tay, L., K.T. Goh and Y.S. Lim. 1994. Vibrio cholerae 0139 'Bengal' in Singapore. J. Trop. Med. Hyg. 97: 317-320.

31) Valdis A.D. and B. Lawrence. 1986. Stable transformation of the cyanobacterium Synechocystis sp. PCC 6803 induced by UV irradiation. J. Bacteriol. 165: 964-971. 